\title{
Lire le lieu pour dire la ville. Florentin : une mise en perspective d'un quartier de Tel Aviv dans la mondialisation (2005-2009)
}

Thèse soutenue par Caroline Rozenholc le 21 mai 2010 à l'Université de Poitiers

\section{Caroline Rozenholc}

\section{OpenEdition}

\section{Journals}

\section{Édition électronique}

URL : https://journals.openedition.org/e-migrinter/1323

DOI : 10.4000/e-migrinter.1323

ISSN : 1961-9685

\section{Éditeur}

UMR 7301 - Migrinter

Édition imprimée

Date de publication : 15 octobre 2010

Pagination : $90-93$

ISSN : 1961-9685

\section{Référence électronique}

Caroline Rozenholc, «Lire le lieu pour dire la ville. Florentin : une mise en perspective d'un quartier de Tel Aviv dans la mondialisation (2005-2009) », e-Migrinter [En ligne], 6 | 2010, mis en ligne le 22 août 2019, consulté le 20 mai 2021. URL : http://journals.openedition.org/e-migrinter/1323 ; DOI : https:// doi.org/10.4000/e-migrinter.1323 


\title{
Lire le lieu pour dire la ville. Florentin : une mise en perspective d'un quartier de Tel Aviv dans la mondialisation (2005-2009)
}

\author{
Thèse soutenue par Caroline Rozenholc le 21 mai 2010 à l'Université de Poitiers
}

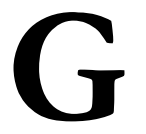

énie du lieu et spatialisation des identités : une problématique remobilisée

La mondialisation est souvent évoquée comme une ère de déstabilisation, d'accélération et de désaffiliation territoriale. Elle semble en effet faire se multiplier ce que M. Augé (1992) avait appelé des «nonlieux », des lieux dépourvus de toute valeur identificatoire, sans âme et sans portée sociale. Toutefois, alors même que l'équation nous liant à certains lieux est remise en mouvement par la part qu'y occupent les mobilités, les modalités de l'appropriation spatiale demeurent une problématique géographique centrale. Ce rapport de la société à son espace fonde véritablement la géographie sans, pour autant, avoir pu être jamais épuisé. A travers ce travail de thèse, il s'agit donc de réinterroger le sens du lieu dans le contexte urbain et c'est le quartier de Florentin, à Tel Aviv, qui constitue l'espace d'où saisir les réarrangements induits par la mondialisation de la ville et du pays. Un peu à la marge d'une ville au dynamisme saisissant, le quartier de Florentin fonctionne dans cette recherche comme un prisme à travers lequel déchiffrer le quotidien d'une société israélienne en pleine transformation. Pour saisir les implications locales de dynamiques mondiales et le jeu d'échelles entre local et global dans un lieu défini, chacune des trois parties de la thèse Explorations géographiques d'un quartier bistorique, Florentin dans la mondialisation et Entre exotisme et nostalgie. Globalisation d'un quartier " authentique 》- décline un aspect du quartier de Florentin, festif, rude, résidentiel et industriel, commercial et de loisirs. Les particularités du quartier de Florentin, tout à la fois singulier dans le paysage de la ville et particulièrement représentatif de l'histoire politique et des mouvements d'immigration qui ont fait Tel Aviv, nous informent en réalité sur les dynamiques à l'œuvre dans l'ensemble stratifié que forme la société israélienne et permettent également de relire l'histoire de la ville.

\section{Identités et identifications : enjeux d'une réflexion située dans un quartier de Tel Aviv}

En effet, l'étude du quartier nous plonge au cœur du développement de Tel Aviv comme projet politique au début du $20^{\text {ème }}$ siècle. Ce travail de lecture du lieu fait alors apparaittre la Tel Aviv, cette «bulle» d'insouciance et de prospérité dans une réalité tourmentée, dans toute la complexité de son contexte régional, bien au-delà de ce que son image uniforme de ville des gratteciels ou du Bauhaus inscrite au patrimoine mondial de l'humanité depuis 2003 ne laisse entendre. Florentin fait contrepoids à la lecture d'une société israélienne repliée d'un côté sur la Tel Aviv globale ouverte sur le monde et de l'autre sur la Jérusalem, locale et religieuse. Créé dans les années 1920 à l'articulation de Tel Aviv et de Jaffa à un moment crucial de l'histoire de la Palestine mandataire, le quartier de Florentin est, dès la création de l'État d'Israël, rapidement abandonné des services publics. Espace de première installation des populations immigrées et poumon industriel et économique de la ville, Florentin se dépeuple et se dégrade toujours plus jusqu'au milieu des années 1990. Il devient, à ce moment là, un espace d'hospitalité réciproque, voire de mobilisation civile et culturelle, pour la jeunesse bohème et les 
travailleurs immigrés, venus d'Europe, d'Asie et d'A frique.

La présence des travailleurs immigrés situe Florentin et les quartiers Sud de Tel Aviv au cœur d'une innovation politique qui, par le biais de la mobilisation civile engagée d'abord depuis ces espaces, a conduit le gouvernement à octroyer la citoyenneté à des enfants de travailleurs immigrés, nés en Israël. Régularisation ponctuelle, en 2005, elle est pourtant la première à élargir le périmètre des affiliations à l'identité israélienne de cette manière. Elle illustre ce que la mondialisation induit de définitions identitaires moins univoques, en particulier pour Israël et pour Tel Aviv. Et puisque ce travail circule entre lieux, entre échelles et entre niveaux de lecture, il faut souligner que c'est la lecture historique de Florentin qui permet de comprendre les distributions socio-économiques au sein de l'agglomération de Tel Aviv Jaffa et la présence, à Florentin de l'ensemble des strates de ceux qui vivent en Israël, citoyens ou étrangers, et de toutes les déclinaisons possibles de ces deux catégories. Dans un contexte national où la population est fragmentée, cette situation est d'ailleurs suffisamment rare pour que la municipalité y propose, par le biais de visites guidées du quartier, un avant-goût d'authenticité et de meltingpot israélien, dans un vieux quartier de Tel Aviv en plein renouveau. Aujourd'hui, après avoir été longtemps écarté de la vision prospère de la ville, Florentin est en pleine fièvre immobilière et se transforme, tant du point de vue de sa population que de son tissu urbain et commercial. Ce quartier d'environ 6000 habitants devient pour certains le lieu où déjouer, avec une certaine nostalgie, les évolutions sociétales très rapides du pays puisque ces transformations se déroulent dans un milieu qui a toujours gardé trace des vagues d'immigration et d'occupations successives du quartier.
Dans des processus détaillés tout au long de la thèse, une des «qualités" particulières de Florentin est de donner à voir que le contenu des espaces ne s'abolit pas au cours du processus social mais qu'il en subsiste toujours «quelque chose » qui se fond en une ambiance spécifique. L'atmosphère de Florentin décrite par différents interlocuteurs, faite de convivialité et de marginalité, de tolérance, de pauvreté et d'embourgeoisement, de superposition de modes de vie somme toute et de pratiques urbaines - industries, logements, commerces, loisirs -, vient d'ailleurs témoigner des réarrangements que produit la mondialisation dans les espaces les plus fluctuants des grandes villes. Dans l'exemple de Florentin, ces fluctuations mettent à jour des lieux jusque là oubliés, enfouis, puis dévoilés par les transformations de la ville. Dans une agglomération partagée en trois zones administratives (Tel Aviv Nord, Centre et Sud) où s'oppose dans des fonctionnalités complémentaires le Nord et le Sud de la ville, Florentin est demeuré à l'écart des grands projets immobiliers et de la transformation du tissu urbain à l'œuvre ailleurs dans la ville. Dans ce sens, le décentrement sans déplacement que permet la constitution de Florentin comme lieu «exotique», à première vue banale, témoigne aussi de la transformation de la centralité de l'État dans la définition des identités. Insister sur Florentin comme lieu de promotion de l'altérité à valeur d'utopie sociale permet de se défaire d'une lecture de l'espace israélien le plus souvent canalisée par la non-résolution du conflit israélopalestinien. La perception de Florentin qui se met en place, comme quartier « exotique » et «authentique» dans la Tel Aviv mondialisée prend aussi sa source dans cette capacité à garder traces d'autres moments de la ville et du pays. L'intérêt de Florentin, pour le chercheur, est d'ailleurs de laisser affleurer, sous des formes plus ou moins explicites, une multitude de lieux et de temporalités accumulées dans la géohistoire particulière du quartier et traduite par le 
rythme qui accompagne les dernières rénovations et l'aura de Florentin.

\section{Méthode pour une géographie sensible}

Dans ce travail, les propos recueillis au cours de près de 80 entretiens sur cinq ans (entre 2005 et 2008) avec des habitants du quartier, des représentants des services de planification de la municipalité et des institutions publiques ou privées actives à Florentin et dans les quartiers Sud, constituent l'unes des principales sources d'information. Les documents d'archive permettent également de mettre la géohistoire du quartier en regard de la place que celui-ci occupe dans la trame de la ville, mais faisant le lien entre coprésence et traces, l'exploration du lieu par la rue est l'une des lignes directrices de ce travail. La « rue» permet de saisir les différentes temporalités, signes et acteurs dans leurs configurations infiniment variables et leurs expressions identitaires quotidiennes. Elle est, par excellence, le lieu de la coprésence et de la confrontation, des aménagements et des tensions. En Israël, et en particulier dans des espaces interstitiels tels que Florentin, c'est un lieu d'observations particulièrement instructif où s'enregistrent présences et traces, affiches et graffitis. Relevés sur les murs du quartier, leur dimension revendicative, mais aussi ludique, est évidente. L'observation et la production de nombreux clichés photographiques permettent alors de rapporter la manière dont les usagers agencent des fragments de sens à travers leurs pratiques dans le quartier de Florentin; un quartier plus que jamais en tension entre le Nord de la ville le plus riche et les quartiers Sud où les services publics sont peu présents.

À travers cette démarche, le positionnement de recherche n'est donc ni de banaliser le lieu ni de conditionner l'approche à un espace unique. Cette méthode d'immersion longue dans le lieu et de multiplication des entrées (entretiens, observation, photographie, participation aux activités sociales, dépouillement des archives) a l'intérêt de pouvoir être développée pour lire d'autres lieux dont, de la même manière, le regard d'un observateur peut dérouler les potentialités et produire une géographie attentive à la multiplication des strates. Dans la mondialisation, celles-ci constituent certains lieux en lieux d'identification forte. Plusieurs exemples développés dans la thèse, en particulier à travers l'analyse de graffitis récurrents, illustrent d'ailleurs parfaitement la puissance d'identification d'un lieu mondialisé. La mondialisation n'est donc pas la dissolution du sens du lieu. Elle est bien plutôt la multiplication de références qu'il porte et des modalités d'identification qu'il permet. En ce sens, l'étude de Florentin est une invitation à ouvrir la réflexion vers d'autres espaces, israéliens ou non. Lire le lieu pour dire la ville, c'est replacer la géographie de la mondialisation sur une approche locale qui prend le temps d'être minutieuse.

\section{Florentin : un terrain fertile}

Focaliser notre recherche sur un quartier de Tel Aviv indique la priorité donnée aux «réarrangements» des lieux produits par la mondialisation sur les questions politiques les plus évidentes dans un espace qui attise les passions identitaires bien au-delà de ses propres frontières. C'est alors de manière détournée, par le caractère situé des phénomènes observés à Florentin, par les manifestations quotidiennes et banales de l'ancrage local et de l'identification au lieu, que l'on approche des questions nationales ou régionales cruciales telles que le débat sur l'identité israélienne et la présence sur le territoire national. Opérant la conjonction d'époques et de passages successifs, ce lieu entre-deux contient en effet en germe tout un faisceau de problématiques et de questionnements qui autorise de monter en généralisation. Le quartier de Florentin témoigne de la démultiplication $\mathrm{du}$ lieu, ouvert mais contenu dans des limites flexibles, et peut être, à ce titre, entrepris comme caisse de 
résonance et de diffraction des registres. Révélant la tension entre proche et lointain, de l'exotisme chez soi et des notions d'authenticité et de nostalgie qui lui sont associées, ce travail circule entre des registres de sens différents, en s'attachant à lire le lieu pour dire la ville contemporaine et celle qui se profile pour demain. Ce travail creuse ainsi le sillon de la réflexion sur le rapport au lieu dans une temporalité mondialisée, d'une territorialité dans son sens le plus large de lien entre individus et entités spatiales du quotidien où le jeu d'échelles entre le local et le global est sans cesse réactivé.
L'intégralité de la thèse est disponible sur le site Hal-SHS à l'adresse suivante :

http://tel.archives-ouvertes.fr/tel$\underline{00496355 / \mathrm{fr} /}$
Caroline Rozenholc Docteure en Géographie MIGRINTER - UMR 6588 CNRS / Université de Poitiers caroline.rozenholc@univ-poitiers.fr 